\title{
DISCUSSION
}

\section{Seismic passive resistance by pseudo-dynamic method}

\author{
D. CHOUDHURY and S. NIMBALKAR (2005). Géotechnique 55, No. 9, 699-702
}

\section{R. Greco, University of Calabria, Italy}

The authors have extended the method proposed by Steedman \& Zeng (1990) to calculate the passive resistance in the presence of horizontal and vertical seismic forces.

This discussion aims to

(a) show that the problem, as formulated by the authors, admits an analytical solution

(b) discuss the effective applicability of the proposed method in calculating the passive resistance

(c) discuss the lateral pressure distribution on the thrust plane $\mathrm{AB}$.

ANALYTICAL SOLUTION OF THE PASSIVE THRUST

The authors suggest obtaining the values of the angle $\alpha$ and time $t$ that minimise the thrust $P_{\mathrm{pe}}$ by means of a trial and error procedure. However, the problem can be solved more effectively analytically, as is shown in the following.

The thrust $P_{\mathrm{pe}}$ depends on the angle $\alpha$ of the failure plane $\mathrm{BC}$ and the time $t$.

$$
\begin{aligned}
P_{\mathrm{pe}}= & \frac{1}{2} \gamma H^{2} \cot \alpha \\
& \times\left[\left(1-k_{\mathrm{v}} \frac{T V_{\mathrm{p}}}{2 \pi H} \frac{m_{2}}{\pi}\right) \frac{\sin \left(\alpha+\phi^{\prime}\right)}{\cos \left(\alpha+\phi^{\prime}+\delta\right)}\right. \\
& \left.-k_{\mathrm{h}} \frac{T V_{\mathrm{s}}}{2 \pi H} \frac{m_{1}}{\pi} \frac{\cos \left(\alpha+\phi^{\prime}\right)}{\cos \left(\alpha+\phi^{\prime}+\delta\right)}\right]
\end{aligned}
$$

The minimum value of $P_{\mathrm{pe}}$ can be obtained by the conditions

$$
\begin{aligned}
& \frac{\partial P_{\mathrm{pe}}}{\partial \alpha}=0 \\
& \frac{\partial P_{\mathrm{pe}}}{\partial t}=0
\end{aligned}
$$

As concerning the condition in equation (14), if we introduce the angle $\theta$, defined as

$$
\tan \theta=\frac{k_{\mathrm{h}} T V_{\mathrm{s}} m_{1}}{2 \pi^{2} H-k_{\mathrm{v}} T V_{\mathrm{p}} m_{2}}
$$

the thrust $P_{\mathrm{pe}}$ becomes

$$
\begin{aligned}
P_{\mathrm{pe}}= & \frac{1}{2} \gamma H^{2} \frac{1}{\cos \theta}\left(1-k_{\mathrm{v}} \frac{T V_{\mathrm{p}}}{2 \pi H} \frac{m_{2}}{\pi}\right) \\
& \times \cot \alpha \frac{\sin \left(\alpha+\phi^{\prime}-\theta\right)}{\cos \left(\alpha+\phi^{\prime}+\delta\right)}
\end{aligned}
$$

which can be rewritten in a form similar to that solved by Mueller-Breslau (1906). Indeed, as in the pseudo-static case (Greco, 2003), making

$$
\phi_{\mathrm{s}}^{\prime}=\phi^{\prime}-\theta
$$

$$
\begin{aligned}
& \delta_{\mathrm{s}}=\delta+\theta \\
& \gamma_{\mathrm{s}}=\gamma \frac{1}{\cos \theta}\left(1-k_{\mathrm{v}} \frac{T V_{\mathrm{p}}}{2 \pi H} \frac{m_{2}}{\pi}\right)
\end{aligned}
$$

it assumes the form

$$
P_{\mathrm{pe}}=\frac{1}{2} \gamma_{\mathrm{s}} H^{2} \cot \alpha \frac{\sin \left(\alpha+\phi_{\mathrm{s}}^{\prime}\right)}{\cos \left(\alpha+\phi_{\mathrm{s}}^{\prime}+\delta_{\mathrm{s}}\right)}
$$

This thrust is minimised when the angle $\alpha$ attains the value

$$
\begin{aligned}
& \alpha=-\phi_{\mathrm{s}}^{\prime}+ \\
& \tan ^{-1}\left[\frac{\tan \phi_{\mathrm{s}}^{\prime}+\sqrt{\frac{\tan \phi_{\mathrm{s}}^{\prime}}{\sin \phi_{\mathrm{s}}^{\prime} \cos \phi_{\mathrm{s}}^{\prime}}\left(1+\frac{\tan \delta_{\mathrm{s}}}{\tan \phi_{\mathrm{s}}^{\prime}}\right)}}{1+\frac{\tan \delta_{\mathrm{s}}}{\sin \phi_{\mathrm{s}}^{\prime} \cos \phi_{\mathrm{s}}^{\prime}}}\right]
\end{aligned}
$$

which depends on the time $t$.

The condition expressed by equation (15) gives the time $t$ when the passive thrust is a minimum. Developing that equation, we have

$$
\frac{t}{T}=\frac{1}{2 \pi} \tan ^{-1}\left[\frac{A_{1}-A_{2} \frac{k_{\mathrm{v}} V_{\mathrm{p}}}{k_{\mathrm{h}} V_{\mathrm{s}}} \tan \left(\alpha+\phi^{\prime}\right)}{B_{1}-B_{2} \frac{k_{\mathrm{v}} V_{\mathrm{p}}}{k_{\mathrm{h}} V_{\mathrm{s}}} \tan \left(\alpha+\phi^{\prime}\right)}\right]+\frac{n}{2}
$$

where

$$
\begin{aligned}
& A_{1}=2 \pi \sin \left(2 \pi \frac{H}{T V_{\mathrm{s}}}\right)+\frac{T V_{s}}{H}\left[\cos \left(2 \pi \frac{H}{T V_{\mathrm{s}}}\right)-1\right] \\
& A_{2}=2 \pi \sin \left(2 \pi \frac{H}{T V_{\mathrm{p}}}\right)+\frac{T V_{\mathrm{p}}}{H}\left[\cos \left(2 \pi \frac{H}{T V_{\mathrm{p}}}\right)-1\right] \\
& B_{1}=2 \pi \cos \left(2 \pi \frac{H}{T V_{\mathrm{s}}}\right)-\frac{T V_{s}}{H} \sin \left(2 \pi \frac{H}{T V_{\mathrm{s}}}\right) \\
& B_{2}=2 \pi \cos \left(2 \pi \frac{H}{T V_{\mathrm{p}}}\right)-\frac{T V_{\mathrm{p}}}{H} \sin \left(2 \pi \frac{H}{T V_{\mathrm{p}}}\right)
\end{aligned}
$$

with $n=1,2,3 \ldots$ In the interval $[0,2 \pi]$, equation (23) has two values, one of which is a minimum and the other is a maximum (Fig. 6). Obviously we are interested in the minimum.

Because the time $t$ in equation (23) depends on $\alpha$, and the angle $\alpha$ in equation (22) depends on $t$, the values of $\alpha$ and $t$, minimising the passive resistance, can be obtained by means of an iterative procedure, which can be articulated as follows.

1. Assume a trial value for $\alpha$ (initially the value related to the condition $\theta=0$ can be assumed).

2. Calculate the coefficients $A_{1}, B_{1}, A_{2}$ and $B_{2}$ using equations (24)-(27).

3 . Through equation (23), calculate the time $t$, corresponding to the minimum, for the current value of $\alpha$.

4. Calculate the coefficients $m_{1}$ and $m_{2}$ using equation (11). 


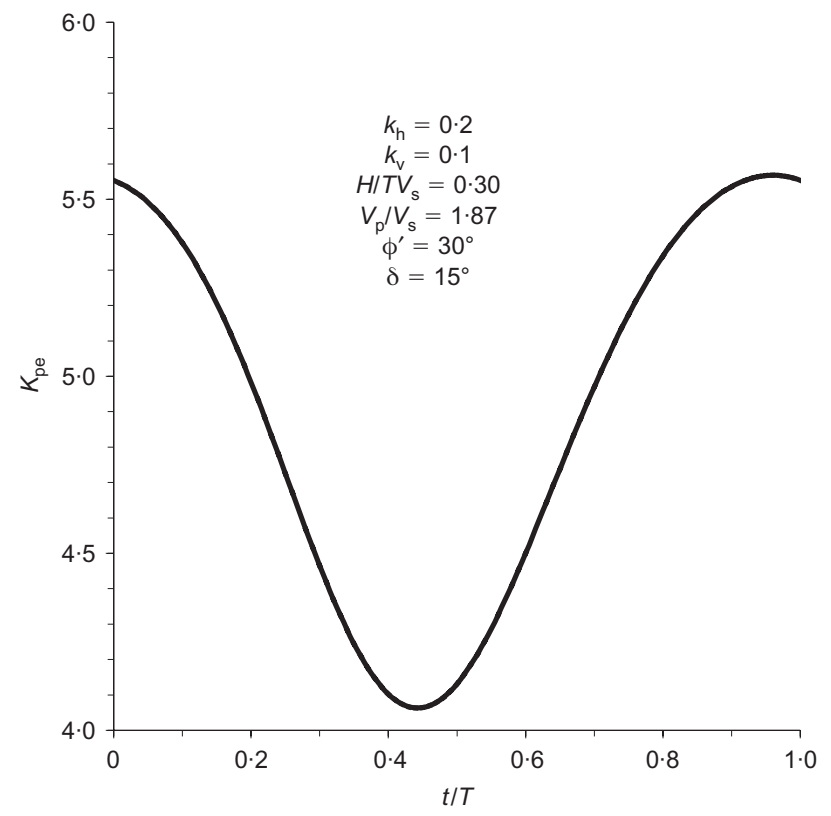

Fig. 6. Pseudo-dynamic passive thrust coefficient $K_{\mathrm{pe}}$ in terms of the ratio $t / T$ for a case of practical interest

5. Calculate the angle $\theta$ with equation (16).

6. Calculate angles $\phi_{\mathrm{s}}^{\prime}$ and $\delta_{\mathrm{s}}$ by means of equations (18) and (19).

7. If the new value of $\alpha$ is sufficiently close to that assumed in step 1:

(a) then go to step 8;

(b) else assume this value of $\alpha$ for a new iteration by starting from step 2.

8. Calculate $\gamma_{\mathrm{s}}$ with equation (20), and $P_{\mathrm{pe}}$ with equation (21).

9. End

This iterative procedure converges quickly.

\section{APPLICABILITY OF THE PROPOSED METHOD}

In the note presented by the authors, the passive thrust is calculated by using the approach of Coulomb (1773), which presupposes a slip surface BC of planar shape. This hypothesis affects the feasibility of the solution considerably. With regard to this, Fig. 7 reports in graphic form the values of passive thrust coefficients, static $\left(K_{\mathrm{p}}\right)$ and pseudo-dynamic $\left(K_{\mathrm{pe}}\right)$, in terms of the ratio $\delta / \phi^{\prime}$. The continuous lines refer to Coulomb's method (the method proposed by the authors is considered as falling within Coulomb's approach); the broken line is obtained by the method of characteristics (Sokolovski, 1960), a method in which the form of the slip surface is not fixed a priori but obtained as part of the solution. As can be seen, Coulomb's method can confidently be used when the ratio $\delta / \phi^{\prime}$ is sufficiently low (generally not over $0 \cdot 3-0 \cdot 4)$. In this case, in fact, the slip surface BC does not differ appreciably from a straight line. For usual values of the ratio $\delta / \phi^{\prime}$ (which are frequently in the range between $1 / 2$ and $2 / 3$ ) the error can became appreciable. This consideration concerns the static passive thrust coefficient $K_{\mathrm{p}}$, but, because the behaviour of $K_{\mathrm{pe}}$ is very similar to that of $K_{\mathrm{p}}$, it is probable that an analogous consideration about the ratio $\delta / \phi^{\prime}$ is also pertinent to $K_{\mathrm{pe}}$.

Moreover, there is another consideration, which it is interesting to take into account. Previously, we transformed the pseudo-dynamic problem into an equivalent static one (and solved it using the solution of Mueller-Breslau), by

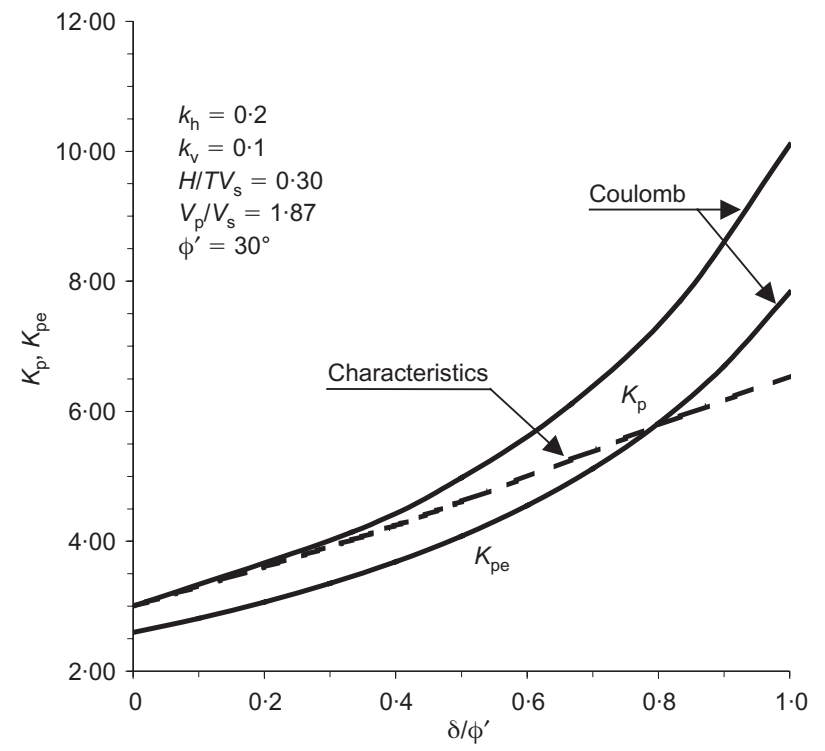

Fig. 7. Static $\left(K_{\mathrm{p}}\right)$ and pseudo-dynamic $\left(K_{\mathrm{pe}}\right)$ passive thrust coefficients in terms of the ratio $\delta / \phi^{\prime}$ for a case of practical interest

substituting the angles $\phi^{\prime}$ and $\delta$ with the angles $\phi_{\mathrm{s}}^{\prime}$ and $\delta_{\mathrm{s}}$ respectively.

As is evident in Fig. 8, relative to a specific case, the value of the ratio $\delta_{\mathrm{s}} / \phi_{\mathrm{s}}^{\prime}$, relative to the value of $t$ minimising $K_{\text {pe }}$, is considerably higher than the corresponding value of the ratio $\delta / \phi^{\prime}$. This seems to suggest the need for a deeper discussion of the question of the applicability of the proposed method, which, however, has the great advantage of a simple formulation and an easy analytical solution.

\section{PRESSURE DISTRIBUTION ON THE WALL}

A rigorous location of the point of application of the thrust requires the evaluation of the lateral pressure distribution $p_{\text {pe }}(z)$. This is generally obtained under the hypothesis that the failure wedge $\mathrm{ABC}$ fails internally along planes, starting from the plane $\mathrm{AB}$ and inclined at angles $\alpha(z)$. The

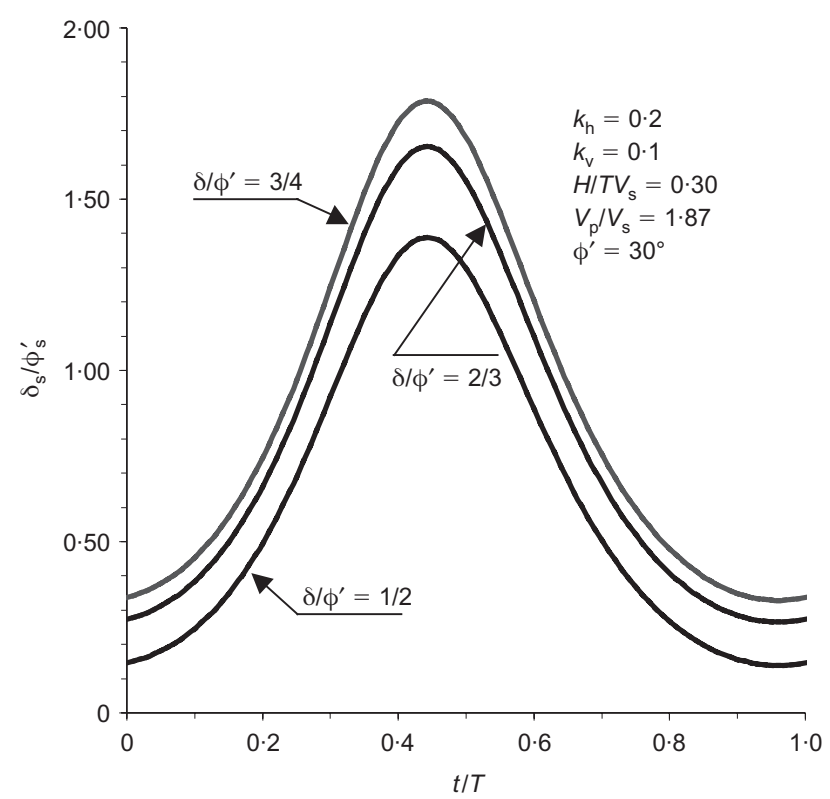

Fig. 8. Ratio $\delta_{\mathrm{s}} / \phi_{\mathrm{s}}^{\prime}$ plotted against the ratio $t / T$ for a case of practical interest 
wedge $\mathrm{ABC}$ thus contains subwedges, having a common vertex $\mathrm{A}$ and being limited by a local failure plane inclined at $\alpha(z)$.

In the method of Coulomb, as generalised by MuellerBreslau, and in the pseudo-static method of Mononobe \& Okabe, these planes are independent of $z$ (are parallel to one another), because the inclination $\alpha$ does not depend on $H$, when we analyse the equilibrium of the wedge ABC. Therefore it does not depend on $z$, when we analyse the equilibrium of a sub-wedge of height $z$, and develop the analysis by substituting $H$ with $z$.

As concerning the pseudo-dynamic analysis, we observe that, as emerges from equations (16) to (22), the angle $\alpha$ depends on $H$ (and, consequently, depends on $z$, when a subwedge $z$ high is considered). In equation (12) of the authors' note, as well as in equation (13) of the paper by Steedman \& Zeng (1990), the pressure distribution on the wall is calculated by differentiating the passive resistance $P_{\text {pe }}$ with respect to the depth $z$, without considering that the angle $\alpha$ is not constant, but varies with $z$.

However, the analytical differentiation of $\alpha(z)$ is complex, and the function $\alpha(z)$ varies a little with $z$ (generally within $\left.3-4^{\circ}\right)$. Therefore the relationship concerning $p_{\text {pe }}(z)$, which must be considered a simplified form, is probably sufficiently approximate for practical applications.

\section{Authors' reply}

The authors are grateful to the discusser for showing interest in their work, and for proposing an alternative approach in which the problem can be solved by an equivalent static analytical approach (Mueller-Breslau, 1906; Greco, 2003).

\section{ANALYTICAL SOLUTION OF THE PASSIVE THRUST}

The discusser has pointed out that the problem can be solved more effectively analytically by transforming the pseudo-dynamic problem into an equivalent static one and solving it by using the solution of Mueller-Breslau as against the pseudo-dynamic method discussed by the authors in their Technical Note, in which the values of the angle $\alpha$ and time $t$ that minimise the thrust $P_{\mathrm{pe}}$ are obtained by means of a trial and error procedure. But the authors would like to emphasise that an effective optimisation technique using the Excel spreadsheet tool Solver has been applied in the present study to minimise the value of seismic passive earth pressure coefficient $\left(K_{\mathrm{pe}}\right)$ with respect to $t / T$ and $\alpha$.

Figures 9 and 10 show the effective use of the optimisation technique used to obtain values of the seismic passive earth pressure coefficients. Again, as compared with the value of seismic passive earth pressure coefficient $\left(K_{\text {pe }}\right)$ reported in Fig. 6 using the alternative analytical approach as suggested by the discusser, the value of seismic passive earth pressure coefficient $\left(K_{\text {pe }}\right)$ reported in Fig. 9 is a minimum. The discusser has reported the minimum value of $K_{\mathrm{pe}}$ as 4.063 for the dimensionless parameter $t / T=0.44$, as shown in Fig. 6. However, the authors have reported the minimum value of $K_{\mathrm{pe}}$ as 3.420 for dimensionless parameter $t / T$ of 0.231 as shown in Fig. 9. Then $K_{\text {pe }}$ is optimised further with respect to failure wedge angle $\alpha$, which yields the value of $K_{\text {pe }}$ as 3.220 for $\alpha=22.5^{\circ}$, as shown in Fig. 10 .

\section{APPLICABILITY OF THE PROPOSED METHOD}

The pseudo-dynamic method of analysis presented in the technical note is an extension to the Mononobe-Okabe method (1926, 1929), which considers the effect of time and phase change in vertically propagating shear and primary

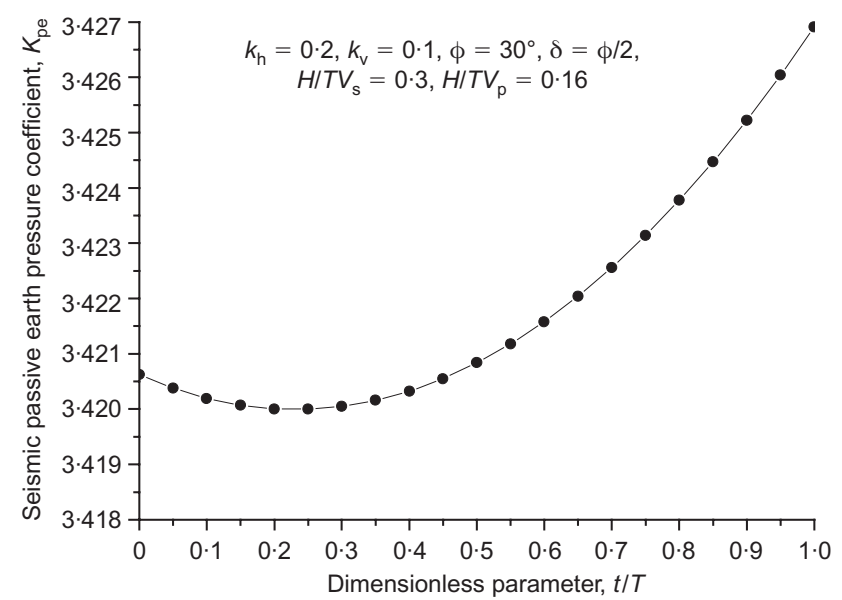

Fig. 9. Seismic passive thrust coefficient $K_{\text {pe }}$ in terms of the ratio $t / T$ for a case of practical interest

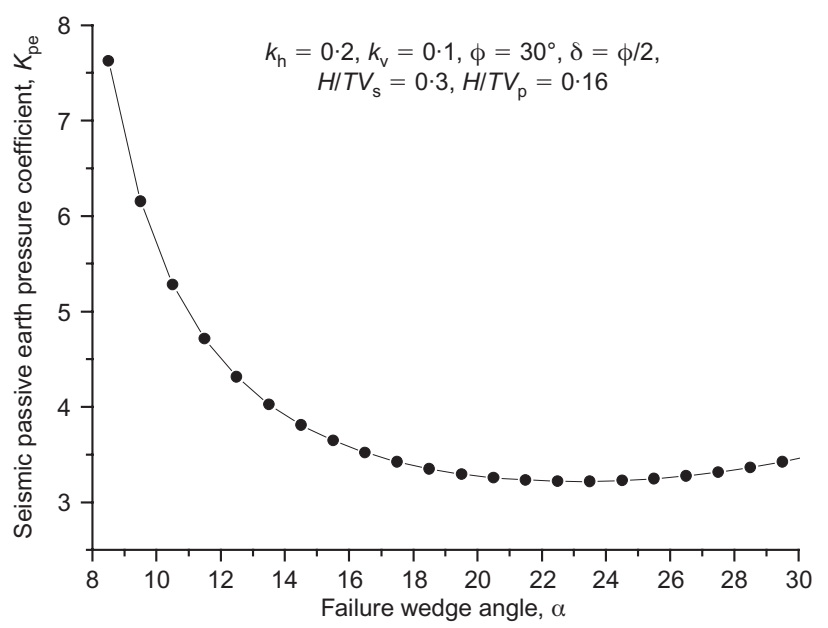

Fig. 10. Seismic passive thrust coefficient $K_{\text {pe }}$ in terms of failure wedge angle $\alpha$ for a case of practical interest

waves through the backfill. The Mononobe-Okabe method, is an extension to Coulomb's (1773) method which assumes the planar rupture surface behind the retaining wall. Thus the pseudo-dynamic method discussed here is also based on the assumption of a planar rupture surface. The authors do admit that this hypothesis affects the feasibility of the solution considerably, that the present solution is applicable when the ratio $\delta / \phi$ is sufficiently low, and that for usual values of the ratio $\delta / \phi$ (which are frequently in the range between $1 / 2$ and $2 / 3$ ) the error can become appreciable. When $\delta / \phi$ is greater than $1 / 2$ the failure surface becomes curved (Kramer, 1996) and the present pseudo-dynamic method, which supposes a slip surface BC of planar shape, cannot be applied. However, the assumption of a planar rupture surface is made in the present pseudo-dynamic method of analysis to avoid further complicating the problem. The assumption of a planar rupture surface for seismic passive earth pressure has also considered by previous researchers (e.g. Mononobe \& Okabe, 1926, 1929—-see Kramer, 1996; Davies et al., 1986).

\section{PRESSURE DISTRIBUTION ON THE WALL}

The discusser has rightly pointed out that in the method of Coulomb (1773) and in the pseudo-static method of Mononobe \& Okabe $(1926,1929)$, failure planes are inde- 
pendent of $z$ and are parallel to one another, because the inclination $\alpha$ does not depend on $H$ when we analyse the equilibrium of the wedge ABC. Therefore it does not depend on $z$, when we analyse the equilibrium of a sub-wedge of height $z$, and develop the analysis by substituting $H$ with $z$. But again, according to the alternative approach suggested by the author (equations (16) to (22)), it is evident that the angle $\alpha$ depends on $H$ (and, consequently, depends on $z$, when a subwedge of height $z$ is considered).

The discusser has pointed out that in equation (12) of the authors' note, and equation (13) of the paper by Steedman \& Zeng (1990), the pressure distribution on the wall is calculated by differentiating the total passive resistance $P_{\mathrm{pe}}$ with respect to the depth $z$, without considering that the angle $\alpha$ is not constant, but varies with $z$.

Again, the discusser has pointed out that the analytical differentiation of $\alpha(z)$ is complex, that the function $\alpha(z)$ varies a little with $z$ (generally within $3-4^{\circ}$ ), and therefore that the relationship concerning $P_{\mathrm{pe}}(z)$, which must be considered in a simplified form, is probably sufficiently approximate for practical applications.

The authors do not agree with this point raised by the discusser, and have found that, in the pseudo-dynamic method of analysis, the failure wedge angle $\alpha$ is constant and does not vary with the height $H$ of the retaining wall.
The value of the failure wedge angle $\alpha$ is selected so as to get the minimum value of seismic passive earth pressure coefficient $\left(K_{\text {pe }}\right)$ and is constant for a given retaining wall of height $H$.

The authors also would like to point out, in the context of the above discussion, that, because the failure wedge angle $\alpha$ does not vary with the height $H$ of the retaining wall and thus consequently does not vary with $z$, the pressure distribution on the wall calculated by differentiating the passive resistance $P_{\text {pe }}$ with respect to the depth $z$ will not be affected by variation of the failure wedge angle $\alpha$. (See equation (12) of the technical note, and equation (13) of the paper by Steedman \& Zeng (1990)).

\section{REFERENCES}

Coulomb, C. A. (1773). Essai sur une application des règles de maximis et minimis a quelques problèmes de statique relatifs a l'architecture. Mémoires de savants étrangers de l'Académie des Sciences de Paris, 7.

Greco, V. R. (2003). Pseudo static analysis for earth thrust computations. Soils Found. 43, No. 2, 135-138.

Mueller-Breslau, H. (1906). Erddruck auf Stützmanern. Stuttgart: Alfred Kröner Verlag (in German).

Sokolovski, V. (1960). Statics of soil media. London: Butterworth. 